\title{
A Case of Goodpasture's Syndrome Associated with Anti-Myeloperoxidase Antibodies
}

\author{
Yoshihiro Arimura, Shinobu Minoshima, Yasushi KamiYa, \\ Kimimasa Nakabayashi, Kiyoshi Kitamoto and Toshihiko Nagasawa
}

\begin{abstract}
A case of Goodpasture's syndrome with anti-myeloperoxidase (MPO) antibodies is reported. Histological examination revealed crescentic glomerulonephritis and alveolar hemorrhage with linear deposition of IgG along the glomerular capillary walls and alveolar capillary walls by immunofluorescence microscopy. Not only anti-glomerular basement membrane (GBM) antibodies but also anti-MPO antibodies, an anti-neutrophil cytoplasmic antibody, were simultaneously detected in the serum. Although it is generally accepted that crescentic glomerulonephritis in Goodpasture's syndrome is mediated by anti-GBM antibodies, this case suggested that anti-MPO antibodies might also participate in the pathogenesis of crescentic glomerulonephritis and probably alveolar hemorrhage of Goodpasture's syndrome, especially with vasculitis.
\end{abstract}

(Internal Medicine 31: 239-243, 1992)

Key words: crescentic glomerulonephritis, anti-neutrophil cytoplasmic antibodies

\section{Introduction}

At present, crescentic glomerulonephritis (CRGN) is classified into three groups on the basis of immunofluorescence. These include linear immune deposits (anti-GBM CRGN), granular immune deposits (immune complex CRGN) and no immune deposits (pauci-immune CRGN) (1). Goodpasture's syndrome is characterized by CRGN and alveolar hemorrhage with linear deposition of $\operatorname{IgG}$ along the glomerular and alveolar basement membranes. It is generally accepted that CRGN in Goodpasture's syndrome is mediated by circulating anti-glomerular basement membrane (GBM) antibodies $(2-3)$.

Anti-neutrophil cytoplasmic antibodies (ANCA) have recently been detected in patients with pauciimmune CRGN, such as idiopathic CRGN and CRGN with systemic vasculitis (4-8). ANCA are divided into two subsets, perinuclear pattern ANCA (p-ANCA) and diffuse cytoplasmic pattern ANCA (c-ANCA), by indirect immunofluorescence of alcohol-fixed human neutrophils. It is known that one p-ANCA is the antibody to myeloperoxidase (MPO) in the neutrophil cytoplasm (5). It has been reported that ANCA, especially anti-
MPO antibodies, might play an important role in the pathogenesis of pauci-immune CRGN (5-7).

In this paper, we report a case of Goodpasture's syndrome associated with anti-MPO antibodies. To our knowledge, there have been only a few previous reports of Goodpasture's syndrome with anti-MPO antibodies $(9-12)$. The present case suggested that not only antiGBM antibodies, but also anti-MPO antibodies may participate in the pathogenesis of CRGN in Goodpasture's syndrome, especially with vasculitis.

\section{Methods}

Anti-GBM antibodies were detected by radioimmunoassay utilizing radiolabeled human GBM antigens isolated from the noncollagenous glycoproteins of the GBM remaining after collagenase digestion. p-ANCA and c-ANCA were detected by indirect immunofluorescence assay (IIF). The method of van der Woude et al (13) was used for IIF. Anti-MPO antibodies were detected by enzyme-linked immunosorbent assay (ELISA) (normal range, $<10$ ELISA unit $/ \mathrm{ml}$ ) using microplates (BioCarb Diagnostics, Lund, Sweden) coated with myeloperoxidase separated from granulocytes. c-ANCA

From The First Department of Internal Medicine, School of Medicine, Kyorin University, Tokyo

Received for publication October 30, 1990; Accepted for publication June 13, 1991

Reprint requests should be addressed to Yoshihiro Arimura, MD, The First Department of Internal Medicine, School of Medicine, Kyorin University, 6-20-2 Shinkawa, Mitaka, Japan 
were detected by using microplate (BioCarb Diagnostics) coated with $29 \mathrm{kd}$ serin protease isolatd from alfa granules of human granulocytes.

\section{Patient}

A 61-year-old male was admitted to a local hospital because of exertional dyspnea on January 12, 1984. He was a smoker ( 40 cigarettes per day) for $30 \mathrm{yr}$, had a history of pulmonary tuberculosis at the age of 31 and had repeated episodes of upper and lower respiratory tract infection. He had no history of Raynaud's phenomenon, hair loss, facial erythema or polyarthralgia suggesting collagen disease. On admission, the chest X-ray disclosed pulmonary fibrosis and bilateral pleural thickening. Laboratory examination revealed a normal urinalysis and normal BUN and serum creatinine ( $\mathrm{S}-\mathrm{Cr}$ ) levels. Hemoptysis, proteinuria and increased BUN and S-Cr were first noted in April 1984. He was transferred to our hospital because of progressively worsening renal failure and respiratory failure on July 16, 1984.

On admission, he was $164 \mathrm{~cm}$ in height and weighed $46.5 \mathrm{~kg}$. Facial and extremity edema was present. His blood pressure was $120 / 80 \mathrm{mmHg}$, and his pulse rate was $106 /$ min and regular. On ausculation, coarse crackles

Table 1. Laboratory Data on Admission

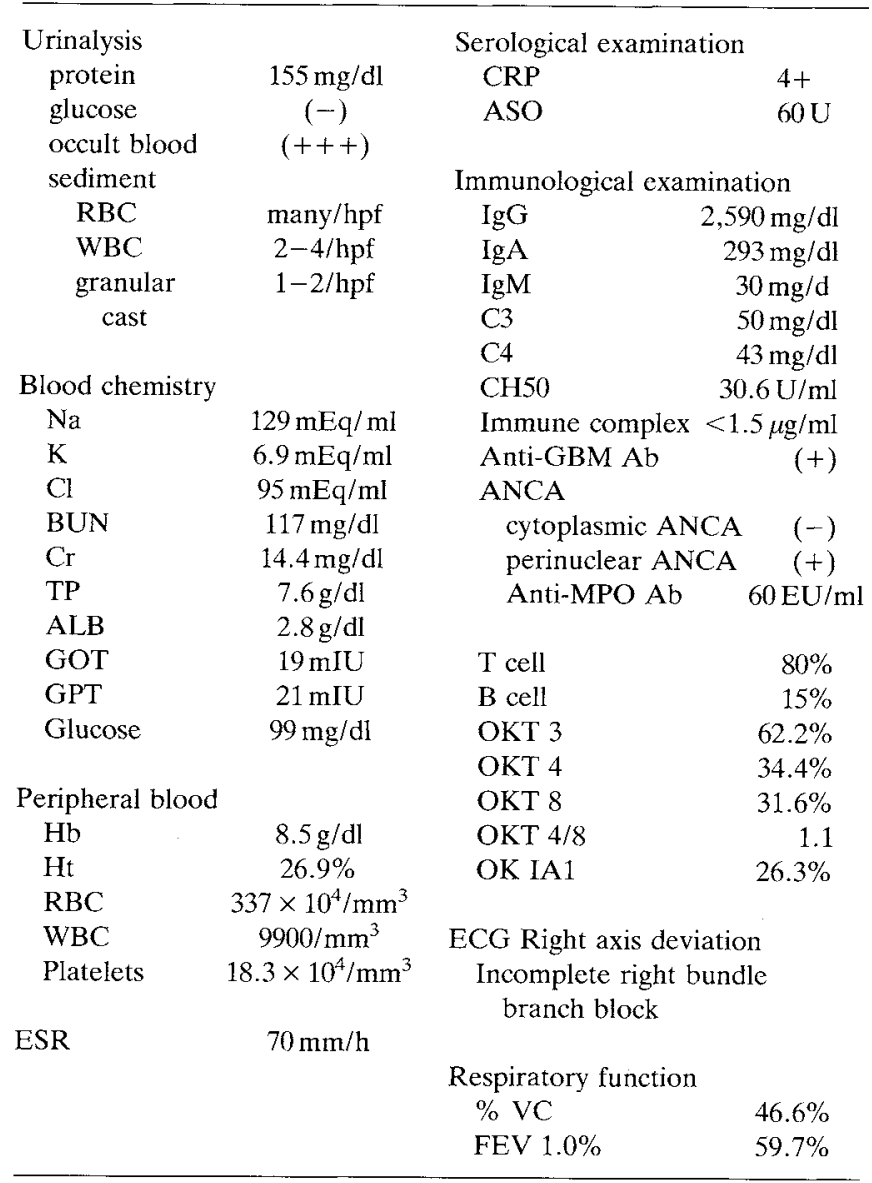

were audible in the right lung and the second pulmonary heart sound was accentuated. Laboratory findings are shown in Table 1 . Urinary protein was $155 \mathrm{mg} / \mathrm{dl}$, urinary sugar was negative and urinary sediment had numerous dysmorphic blood cells and 1 to 2 granular casts/HPF. Laboratory data included BUN $117 \mathrm{mg} / \mathrm{dl}$, $\mathrm{S}-\mathrm{Cr} 14.4 \mathrm{mg} / \mathrm{dl}$, and potassium $6.5 \mathrm{meq} / \mathrm{l}$. WBC was $9,900 / \mathrm{mm}^{3}$, hematocrit $26.9 \%$, and c-reactire protein $4+$, C3 was $50 \mathrm{mg} / \mathrm{dl}$, and $\mathrm{C} 4$ and $\mathrm{CH} 50$ levels were within normal range. Circulating immune complexes were not present but anti-GBM antibodies were positive. P-ANCA were detected by IIF and anti-MPO antibodies were detected at $60 \mathrm{ELISA}$ unit/ml. Anti-nuclear antibodies were positive $(1: 160)$ in a speckled pattern. Anti-DNA antibodies, anti-RNP antibodies, anti-SM antibodies and rheumatoid factors were not detected. Blood, sputum and urine cultures were negative. A chest $\mathrm{X}$-ray showed a reticular shadow in the right midzone, pulmonary fibrosis and pleural thickening as shown in Fig. 1.

From these clinical and laboratory findings, a diagnosis of rapidly progressive glomerulonephritis with pulmonary hemorrhage was made. As shown in Fig. 2, the patient was started on prednisolone $(60 \mathrm{mg} /$ day $)$ and hemodialysis. He died of respiratory failure 3 months after admission.

Autopsy revealed CRGN and alveolar hemorrhage



Fig. 1. Chest roentgenogram showing both pleural thickening and reticular shadows in the right lung. 
ANCA in Goodpasture's Syndrome

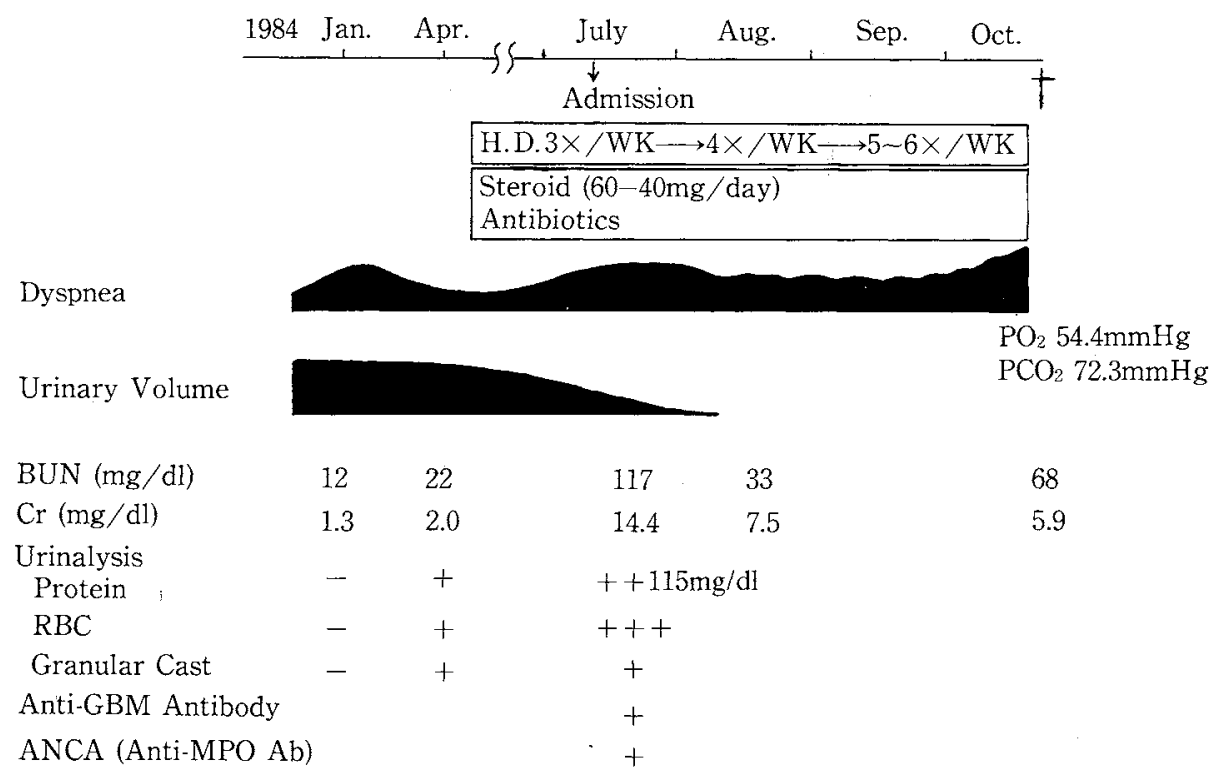

Fig. 2. Clinical course (K.T. 61 years old, male).

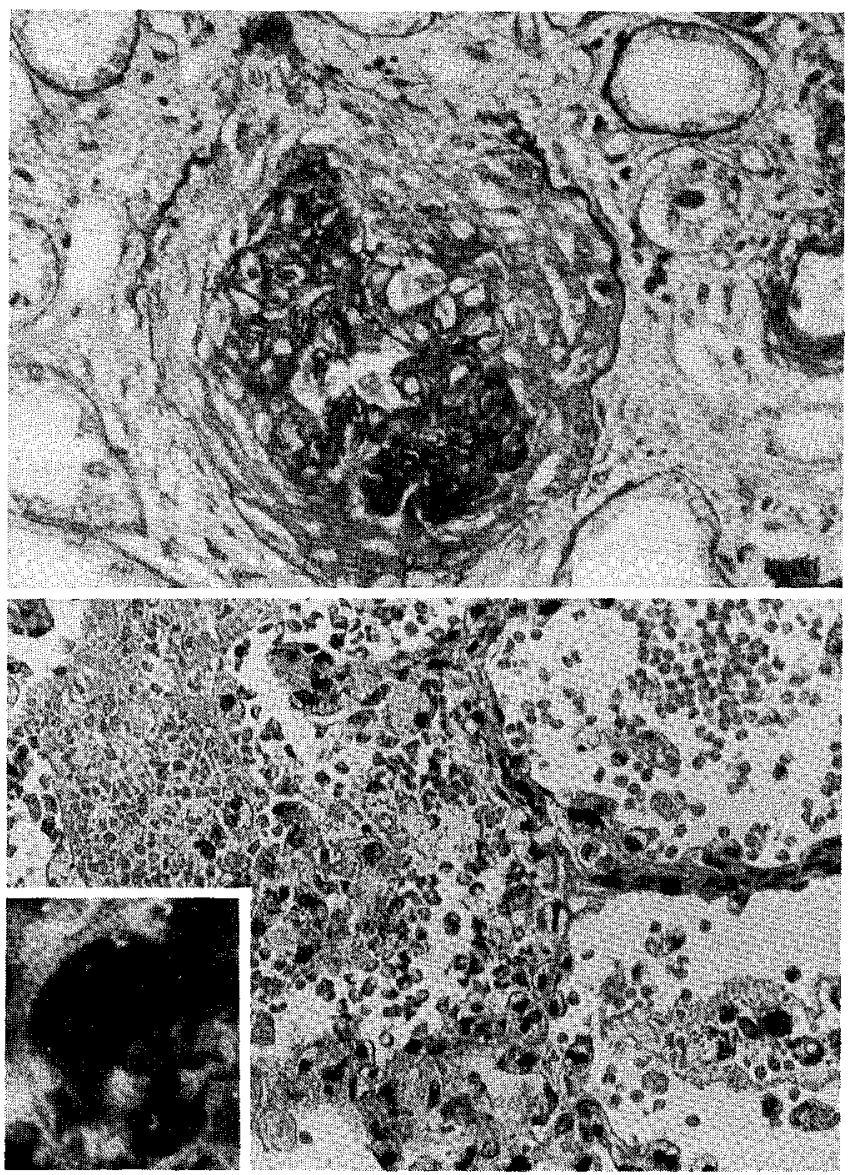

Fig. 3. A) Light microscopy of a kidney specimen showing marked fibro-cellular crescent formation (PAS, $\times 200)$. B) Light microscopy showing massive hemorrhage in the alveolar lumens (H.E. $\times 200$ ). C) Immunofluorescent micrography showing linear deposition of IgG along the alveolar capillary wall $(\times 150)$. A

$$
\frac{A}{C \cdot B}
$$

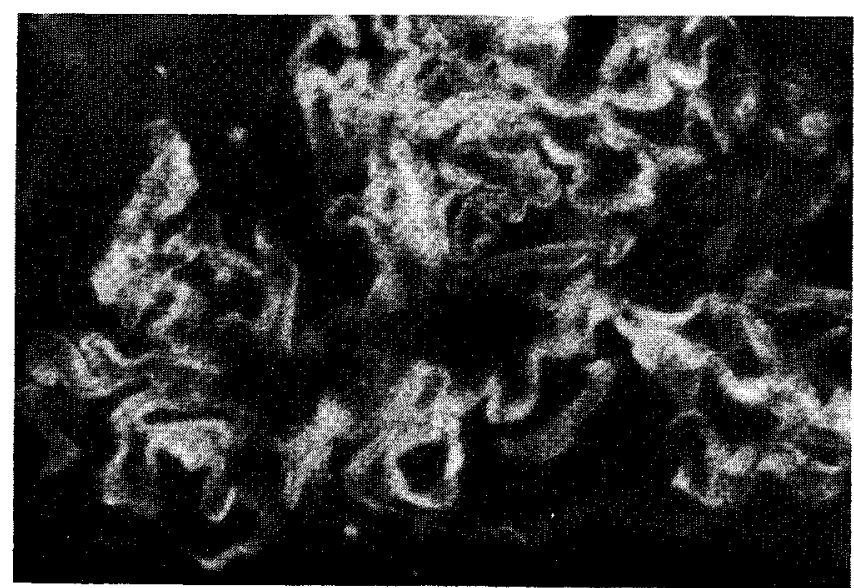

Fig. 4. Immunoffuorescent micrography showing linear deposition of $\operatorname{IgG}$ along the glomerular capillary wall $(\times 400)$.

as shown in Fig. 3. Immunofluorescence studies demonstrated a linear deposition of $\operatorname{IgG}$ along the glomerular capillary wall and the alveolar capillary wall as shown in Figs. 3-C and 4. Systemic vasculitis, pneumonia, active tuberculosis, left atrial myxoma, leptospirosis or collagen diseases were not found.

\section{Discussion}

Goodpasture's syndrome is a clinical disorder of unknown cause characterized by rapidly progressive glomerulonephritis and pulmonary hemorrhage. The pathogenesis of Goodpasture's syndrome appears to involve circulating antibodies directed toward the glomerular basement membrane and alveolar basement membrane. Therefore, the diagnosis of Goodpasture's syndrome is now based on clinical, histological and 
immunological findings. In Japan, Goodpasture's syndrome is very rare; there have been only a few reports which fulfill the criteria. The present case showed rapidly progressive glomerulonephritis with pulmonary hemorrhage and crescentic glomerulonephritis with alveolar hemorrhage. Immunological studies revealed linear deposition of $\operatorname{IgG}$ along the glomerular capillary wall and alveolar capillary wall and circulating anti-GBM antibodies were detected. This evidence indicates findings characteristic of Goodpasture's syndrome.

The most noteworthy feature in the present case was the simultaneous presence of both circulating anti-MPO antibodies, one of the ANCA subsets, and circulating anti-GBM antibodies.

ANCA were originally described in segmental necrotizing glomerulonephritis and have subsequently been detected in idiopathic CRGN and systemic vasculitis, such as Wegener's granulomatosis and microscopic polyarteritis $(4-8,13)$. In Japan, ANCA were first described by us (8) in patients with Wegener's granulomatosis in 1988. CRGN is frequently seen with systemic vasculitis, and both CRGN with systemic vasculitis and idiopathic CRGN belong to pauci-immune CRGN with ANCA. Balow (14) and Savage et al (15) reported that idiopathic CRGN is related to the spectrum of vasculitis known as microscopic polyarteritis, since systemic vasculitis might begin as kidney limited polyarteritis, and they suggested that there is a common pathogenesis between systemic vasculitis and idiopathic CRGN. Falk and Jennette (5) reported that ANCA, especially anti-MPO antibodies, are related to idiopathic CRGN as renal-limited polyarteritis, rather than extra-renal systemic vasculitis, and that C-ANCA are related to lung and sinus involvement. It is reported that the sensitivity and specificity of anti-MPO antibodies for idiopathic CRGN are $75 \%$ and $92 \%$, respectively (6). We (7) reported 11 cases with anti-MPO antibodies, 7 cases were CRGN and 4 cases were glomerulonephritis with a moderate degree of crescent formation. Six of the 7 CRGN cases with anti-MPO antibodies were pauci-immune CRGN. Furthermore, 3 of the 6 cases of pauci-immune CRGN were associated with pulmonary hemorrhage. Therefore, it is suggested that ANCA, especially anti-MPO antibodies, may play an important pathogenic role in pauci-immune $\mathrm{CRGN}$ as renal vasculitis and probably pulmonary hemorrhage.

In the present case, anti-MPO antibodies were detected, but C-ANCA were negative. Also, systemic vasculitis was not found at autopsy.

ANCA have been detected in only a few patients without vasculitis or CRGN, including atrial myxoma, pneumonia, leptospirosis, active tuberculosis and collagen diseases (16-18). These infectious and collagen diseases were not found on clinical or pathological examination in the present case. These findings suggest that anti- MPO antibodies may have played a role in the pathogenesis of
CRGN as renal vasculitis in the present patient with Goodpasture's syndrome.

Although there have been few reports of patients with both ANCA and anti-GBM antibodies, Pusey and Lockwood (9) reported 20 patients with both antibodies in 890 patients with rapidly progressive glomerulonephritis and Jayne et al (10) reported that ANCA are found in up to $30 \%$ of patients with anti-GBM disease. Furthermore, O'Donoghue et al (11) reported 3 patients with both anti-GBM antibodies and c-ANCA associated with vasculitis, and they suggested that $\mathrm{c}-\mathrm{ANCA}$ may identify a distinct subset of patients with anti-GBM disease. Vanhille et al (12) reported a patient who initially presented with anti-GBM glomerulonephritis and developed vasculitis with anti-MPO antibodies. Although ANCA were not measured, Goodpasture (19), Stanton and Tange (20), Wu et al (2) and Kondo et al (3) all reported Goodpasture's syndrome associated with systemic vasculitis.

Anti-nuclear antibodies were detected in the present patient, but collagen diseases and other diseases usually associated with anti-nuclear antibodies were not found on clinical and pathological examination. Although the function of cellular immunity and genetic factors was not examined, an immunological disorder producing autoantibodies, such as anti-nuclear antibodies and antiMPO antibodies, might have been present in this case.

Although the pathogenic role of anti-MPO antibodies in CRGN remains unknown, Falk et al (21) reported that anti-MPO antibodies activate neutrophils and induce oxygen radical release and primary granule degranulation. It is reported that MPO exists in the primary granule as a neutrophil cationic enzyme which reacts with glomerular polyanion on the glomerular capillary wall, and MPO causes glomerular injury by reacting with $\mathrm{H}_{2} \mathrm{O}_{2}$, which is one of the reactive oxygen species derived from activated neutrophils $(22,23)$. MPO on the glomerular capillary wall might be related to basement membrane damage resulting in the rupture of the GBM via neutrophil activation by anti-MPO antibodies. The mechanism of the simultaneous presence of both anti-MPO antibodies and anti-GBM antibodies is not known. Since cross reactivity of these autoantibodies is not found, there may be some common immunological disorder of cellular immunity producing these autoantibodies.

It was concluded that not only anti-GBM antibodies but also anti-MPO antibodies might participate in the pathogenesis of CRGN and probably alveolar hemorrhage in Goodpasture's syndrome, especially that associated with vasculitis.

Acknowledgements: We wish to thank Mr. Kazuhiro Fukutomi for his technical assistance.

\section{References}

1) Salant DJ. Immunopathogenesis of crescentic glomerulonephritis 


\section{ANCA in Goodpasture's Syndrome}

and lung purpura. Kidney Int 32: 408, 1987.

2) Wu MJ, Rajaram R, Shelp WD, Beirne GJ, Burkholder PM. Vasculitis in Goodpasture's syndrome. Arch Pathol Lab Med 104: $300,1980$.

3) Kondo N, Tateno M, Yamaguchi J, et al. Immunopathological studies of an autopsy case with Goodpasture's syndrome and systemic necrotizing angitis. Acta Pathol Jpn 36: 595, 1986.

4) Davies DJ, Moran JE, Niall JF, Ryan GB. Segmental necrotising glomerulonephritis with antincutrophil antibody: Possible arbovirus aetiology? Br Med J 285: 606, 1982.

5) Falk RJ, Jennette JC. Anti-neutrophil cytoplasmic autoantibodies with specificity for myelopcroxidase in patients with systemic vasculitis and idiopathic necrotizing and crescentic glomerulonephritis. N Engl J Med 318: 1651, 1988.

6) Cohen Tervaert JW, Goldschmeding R, Elema JD, et al. Autoantibodies against myeloid lysosomal enzymes in crescentic glomerulonephritis. Kidney Int 37: 799, 1990.

7) Arimura $Y$, Nohmi K, Tanaka U, Nagasawa T. Clinical investigation of antimyeloperoxidase antibodies in patients with glomerulonephritis associated with crescent. Jpn J Nephrol (in Japanese, English Abstract) 32: 1087, 1990.

8) Yoshida $M$, Nagasawa $T$. Autoantibodies against neutrophil cytoplasma in systemic vasculitis especially in Wcgener's granulomatosis. Igakunoayumi 145: 649, 1988. (in Japanese, English Abstract)

9) Pusey CD, Lockwood CM. Autoimmunity in rapidly progressive glomerulonephritis. Kidney Int 35: 929, 1989.

10) Jayne DRW, Marshall PD, Joncs SJ, Lockwood CM. Autoantibodies to GBM and neutrophil cytoplasm in rapidly progressive glomerulonephritis. Kidney Int 37: 965, 1990.

11) O'Donoghue DJ, Short CD, Brenchley PEC, Lawler W, Ballardie FW. Sequential development of systemic vasculitis with antineutrophil cytoplasmic antibodies complicating anti-glomerular basement membrane disease. Clin Nephrol 32: 251, 1989.

12) Vanhille $P$, Noel LH, Reumaux D, Fleury D, Lamaitre $V$, Gobert P. Late emergence of systemic vasculitis with antineutrophil cytoplasmic antibodies in a dialyzed patient with anti- golmcrular basement glomeruloncphritis. Clin Nephrol 33: 257, 1990.

13) Woude FJ, Rasmussen N, Lobatto S, et al. Autoantibodies against neutrophils and monocyte: Tool for diagnosis and marker of disease activity in Wegener's granulomatosis. Lancet 1: 425, 1985.

14) Balow JE. Renal vasculitis (Nephrology Forum). Kidney Int 27: 954, 1985.

15) Savage COS, Winearls CG, Evans DJ, et al. Microscopic polyarteritis: Presentation, pathology and prognosis. Q J Mcd 56: $467,1985$.

16) Clerck LS, Offer JF, Smolders WA, et al. Pitfalls with antincutrophil cytoplasmic antibodics (ANCA). Clin Rheumatol 8: $512,1989$.

17) Savage COS, Winearls CG, Jones S, Marshall PD. Prospective study of radioimmunoassay for antibodies against neutrophil cytoplasm in diagnosis of systemic vasculitis. Lancet 1: 1389, 1987.

18) Savige JA, Yeung SP, Davies DJ. Anti-ncutrophil cytoplasmic antibodies associated with atrial myxoma. Am J Med 85: 755, 1988.

19) Goodpasture EW. The significance of certain pulmonary lesions in relation to the etiology of influenza. Am J Med Sci 158: 863, 1919.

20) Stanton MC, Tange JD. Goodpasture's syndrome. Aust Ann Med 7: 132, 1958.

21) Falk RJ, Terrell RS, Charles LA, Jennette JC. Anti-neutrophil cytoplasmic autoantibodies induce neutrophils to degranulate and produce oxygen radicals in vitro. Proc Natl Acad Sci 87: 4115, 1990.

22) Johnson RJ, Couser WG, Chi EY, Alder S, Klebanoff SJ. New mechanism for glomerular injury myeloperoxidase-hydrogen peroxidase-halide system. J Clin Invest 79: 1379, 1987.

23) Johnson RJ, Guggenheim SJ, Klebanoff SJ, et al. Morphologic correlates of glomerular oxidant injury induced by the myeloperoxidasc-hydrogen peroxide-halide system of the neutrophil. Lab Invest 5: 294, 1988. 